\title{
Dynamical noncommutative quantum mechanics
}

\author{
S. A. Alavi, S. Abbaspour \\ Department of Physics, Hakim Sabzevari University, P. O. Box \\ 397, Sabzevar, Iran. \\ s.alavi@hsu.ac.ir ; alialavi@fastmail.us
}

\begin{abstract}
We study some basic and interesting quantum mechanical systems in dynamical noncommutative spaces in which the space- space commutation relations are position dependent. It is observed that the fundamental objects in the dynamical noncommutative space introduced here are stringlike. We show that the Stark effect can be employed to determine whether the noncommutativity of space is dynamical or non-dynamical. It appears that unlike non-dynamical case there is a fundamental energy $\frac{\tau \hbar^{2}}{m}$ in this dynamical space.
\end{abstract}

\section{Introduction}

The idea of extension of noncommutativity to the coordinates was first suggested by Heisenberg as a possible solution for removing the infinite quantities of field theories. The renewed interest by physics community started to grow after the paper by Seiberg and Witten [1], see also [2]. Noncommutative quantum mechanics has received a wide attention in recent years and many physical problems have been studied in the framework of the noncommutative quantum mechanics.

The noncommutativity of the coordinates can be described by the following commutation relation:

$$
\left[x_{\mu}, x_{\nu}\right]=i \theta_{\mu \nu}
$$

where $\theta_{\mu \nu}$ is an anti-symmetric tensor. The simplest case corresponds to $\theta_{\mu \nu}$ being constant, which we call it non-dynamical or $\theta$-noncommutative spaces. In [3], the authors assumed $\theta_{\mu \nu}$ to be a function of position coordinates.

Recently [4] a generalization to dynamical( position dependent ) noncommutative spaces (DNCS) has been proposed in which the noncommutativity tensor is not constant but is position dependent and the following commutation relations are introduced for a two dimensional dynamical noncommutative space :

$$
\begin{aligned}
& {[X, Y]=i \theta\left(1+\tau Y^{2}\right) \quad ; \quad\left[X, P_{x}\right]=i \hbar\left(1+\tau Y^{2}\right) \quad ; \quad\left[X, P_{y}\right]=2 i \tau Y\left(\theta P_{y}+\hbar X\right)} \\
& {\left[Y, P_{y}\right]=i \hbar\left(1+\tau Y^{2}\right) \quad ; \quad\left[Y, P_{x}\right]=0 \quad ; \quad\left[P_{x}, P_{y}\right]=0}
\end{aligned}
$$


It is worth mentioning that by taking $\tau \rightarrow 0$, we recover the non-dynamical ( $\theta$-noncommutative) commutation relations:

$$
\begin{aligned}
& {\left[x_{0}, y_{0}\right]=i \theta \quad ; \quad\left[x_{0}, p_{x_{0}}\right]=i \hbar \quad ; \quad\left[x_{0}, p_{y_{0}}\right]=0} \\
& {\left[y_{0}, p_{y_{0}}\right]=i \hbar \quad ; \quad\left[y_{0}, p_{x_{0}}\right]=0 \quad ; \quad\left[p_{x_{0}}, p_{y_{0}}\right]=0}
\end{aligned}
$$

The $X$ coordinate and the momentum in $Y$ direction, $P_{y}$ are not Hermitian so the Hamiltonian involving these variables will in general also not be Hermitian. But we may look for a similarity transformation i.e. a Dyson map $\eta O \eta^{-1}=o=o^{\dagger}$ which convert the non-Hermitian system into a Hermitian one. It is shown that the relevant Dyson map is $\eta=\left(1+\tau Y^{2}\right)^{-1 / 2}$, so the new Hermitian variables $x, y, p_{x}$ and $p_{y}$ can be stated in terms of $\theta$-noncommutative space variables as follows [4]:

$$
\begin{aligned}
x=\eta X \eta^{-1} & =\left(1+\tau y_{0}^{2}\right)^{-1 / 2}\left(1+\tau y_{0}^{2}\right) x_{0}\left(1+\tau y_{0}^{2}\right)^{1 / 2} \\
& =\left(1+\tau y_{0}^{2}\right)^{1 / 2} x_{0}\left(1+\tau y_{0}^{2}\right)^{1 / 2} \\
y=\eta Y \eta^{-1} & =\left(1+\tau y_{0}^{2}\right)^{-1 / 2} y_{0}\left(1+\tau y_{0}^{2}\right)^{1 / 2}=y_{0} \\
p_{x}=\eta P_{x} \eta^{-1} & =\left(1+\tau y_{0}^{2}\right)^{-1 / 2} p_{x_{0}}\left(1+\tau y_{0}^{2}\right)^{1 / 2}=p_{x_{0}} \\
p_{y}=\eta P_{y} \eta^{-1} & =\left(1+\tau y_{0}^{2}\right)^{-1 / 2}\left(1+\tau y_{0}^{2}\right) p_{y_{0}}\left(1+\tau y_{0}^{2}\right)^{1 / 2} \\
& =\left(1+\tau y_{0}^{2}\right)^{1 / 2} p_{x_{0}}\left(1+\tau y_{0}^{2}\right)^{1 / 2}
\end{aligned}
$$

where zero index indicates the coordinates in $\theta$-noncommutative spaces.

These Hermitian dynamical noncommutative variables satisfy the following relations:

$$
\begin{aligned}
{[x, y] } & =i \theta\left(1+\tau y^{2}\right) \quad ; \quad\left[x, p_{x}\right]=i \hbar\left(1+\tau y^{2}\right) \quad ; \quad\left[p_{x}, p_{y}\right]=0 \\
{\left[x, p_{y}\right] } & =2 i \tau y\left(\theta p_{y}+\hbar x\right) \quad ; \quad\left[y, p_{x}\right]=0 \quad ; \quad\left[y, p_{y}\right]=i \hbar\left(1+\tau y^{2}\right)
\end{aligned}
$$

Using Bopp-shift one can relate the $\theta$-noncommutative variables to the variables of standard (conventional) commutative space:

$$
x_{i_{0}}=x_{i_{s}}-\frac{\theta_{i j}}{2 \hbar} p_{j_{s}} \quad, \quad p_{i_{0}}=p_{i_{s}} \quad, \quad i, j=x, y
$$

where $\theta_{i j}=\epsilon_{i j k} \theta_{k}$ and we take $\theta_{3}=\theta$ and the rest of the $\theta$-components to zero [7].

An interesting point is that the minimal uncertainty of the coordinate $X$ :

$$
\Delta X_{\text {min }}=\theta \sqrt{\tau} \sqrt{1+\tau\langle Y\rangle_{\rho}^{2}}
$$

leads to a minimal length for $X$ in a simultaneous $X, Y$ measurment, but there is no nonvanishing length in the $Y$-direction [4]. Here $\rho=\eta^{2}=\left(1+\tau Y^{2}\right)^{-1}$, is the metric operator, and $\langle Y\rangle_{\rho}$ is the expectation value of operator $Y$ with respect to this metric.

This means that the fundamental objects in this type of dynamical noncommutative spaces are string like and this is a good motivation to study physics in these spaces. In this paper we study some interesting and important quantum systems in DNCS. 


\section{The harmonic oscillator}

Harmonic oscillator is an important model in many branches of physics. It can be used to illustrate the basic concepts and methods in quantum mechanics. It also has applications in a variety of branches of modern physics including spectroscopy, condensed matter physics, nuclear structure, quantum optics, quantum field theory and statistical mechanics.

The Hamiltonian of a two dimensional harmonic oscillator in DNCS is given by:

$$
H\left(x, y, p_{x}, p_{y}\right)=\frac{p_{x}^{2}+p_{y}^{2}}{2 m}+\frac{1}{2} m \omega^{2}\left(x^{2}+y^{2}\right)
$$

Using Eq.(4), we rewrite the Hamiltonian in terms of $\theta$-noncommutative variables:

$$
\begin{aligned}
h\left(x_{0}, y_{0}, p_{x_{0}}, p_{y_{0}}\right) & =\frac{1}{2 m}\left[p_{x_{0}}^{2}+\left(1+\tau y_{0}^{2}\right)^{1 / 2} p_{y_{0}}\left(1+\tau y_{0}^{2}\right) p_{y_{0}}\left(1+\tau y_{0}^{2}\right)^{1 / 2}\right] \\
& +\frac{1}{2} m \omega^{2}\left[\left(1+\tau y_{0}^{2}\right)^{1 / 2} x_{0}\left(1+\tau y_{0}^{2}\right) x_{0}\left(1+\tau y_{0}^{2}\right)^{1 / 2}+y_{0}^{2}\right]
\end{aligned}
$$

with the help of the Bopp-shift this Hamiltonian can be stated in terms of the standard commutative variables. To the first order in $\theta$ and $\tau$ we have:

$$
\begin{aligned}
H_{h o}\left(x_{s}, y_{s}, p_{x_{s}}, p_{y_{s}}\right) & =\frac{p_{x_{s}}^{2}+p_{y_{s}}^{2}}{2 m}+\frac{1}{2} m \omega^{2}\left(x_{s}^{2}+y_{s}^{2}\right)-\frac{m \omega^{2} \theta}{2 \hbar} L_{z_{s}} \\
& +\frac{\tau}{m} y_{s}^{2} p_{y_{s}}^{2}+m \omega^{2} \tau y_{s}^{2} x_{s}^{2}-2 i \frac{\tau \hbar}{m} y_{s} p_{y_{s}}-\frac{\tau \hbar^{2}}{2 m} \\
& =H_{h o}^{s}+H_{\theta, \tau}
\end{aligned}
$$

where $H_{h o}^{s}$ is the Hamiltonian of harmonic oscillator in commutative spaces. If we set $\tau=0$ we get the Hamiltonian of harmonic oscillator in $\theta$-noncommutative case i.e. Eq.(8) of Ref.[5](we note that in this equation to the first order in $\theta$, the paramete $k=1)$.

Since the noncommutativity parameters $\tau$ and $\theta$ if they are non-zero should be very small, we use perturbation theory to find the spectrum of quantum systems. The perturbation Hamiltonian can be rewritten as follows:

$$
\begin{aligned}
H_{\theta, \tau} & =-\frac{m \omega^{2} \theta}{2 \hbar} L_{z_{s}}+\frac{\tau}{m} y_{s}^{2} p_{y_{s}}^{2}+m \omega^{2} \tau y_{s}^{2} x_{s}^{2}-2 i \frac{\tau \hbar}{m} y_{s} p_{y_{s}}-\frac{\tau \hbar^{2}}{2 m} \\
& =v_{1}+v_{2}+v_{3}+v_{4}+v_{5}
\end{aligned}
$$


The contributions of the different parts of the perturbation Hamiltonian are as follows:

$$
\begin{aligned}
&\left\langle n_{x}, n_{y}\left|v_{1}\right| n_{x}^{\prime}, n_{y}^{\prime}\right\rangle=-\frac{i}{2} m \omega^{2} \theta \sqrt{\left(n_{y}^{\prime}+1\right) n_{x}^{\prime}} \delta_{n_{x}, n_{x}^{\prime}+1} \delta_{n_{y}, n_{y}^{\prime}-1} \\
&+\frac{i}{2} m \omega^{2} \theta \sqrt{\left(n_{x}^{\prime}+1\right) n_{y}^{\prime}} \delta_{n_{x}, n_{x}^{\prime}-1} \delta_{n_{y}, n_{y}^{\prime}+1} \\
&\left\langle n_{x}, n_{y}\left|v_{2}\right| n_{x}^{\prime}, n_{y}^{\prime}\right\rangle=\frac{\tau \hbar^{2}}{4 m}\left(2 n_{y}^{\prime 2}+2 n_{y}^{\prime}-1\right) \delta_{n_{x}, n_{x}^{\prime}} \delta_{n_{y}, n_{y}^{\prime}} \\
&\left\langle n_{x}, n_{y}\left|v_{3}\right| n_{x}^{\prime}, n_{y}^{\prime}\right\rangle=\frac{\tau \hbar^{2}}{4 m}\left[\sqrt{\left(n_{y}^{\prime}-1\right) n_{y}^{\prime}} \delta_{n_{y}, n_{y}^{\prime}-2}+\left(2 n_{y}^{\prime}+1\right) \delta_{n_{y}, n_{y}^{\prime}}\right. \\
&\left.+\sqrt{\left(n_{y}^{\prime}+1\right)\left(n_{y}^{\prime}+2\right)} \delta_{n_{y}, n_{y}^{\prime}+2}\right]\left[\sqrt{\left(n_{x}^{\prime}-1\right) n_{x}^{\prime}} \delta_{n_{x}, n_{x}^{\prime}-2}\right. \\
&\left.+\left(2 n_{x}^{\prime}+1\right) \delta_{n_{x}, n_{x}^{\prime}}+\sqrt{\left(n_{x}^{\prime}+1\right)\left(n_{x}^{\prime}+2\right)} \delta_{n_{x}, n_{x}^{\prime}+2}\right] \\
&\left\langle n_{x}, n_{y}\left|v_{4}\right| n_{x}^{\prime}, n_{y}^{\prime}\right\rangle=\frac{\tau \hbar^{2}}{m} \delta_{n_{x}, n_{x}^{\prime}} \delta_{n_{y}, n_{y}^{\prime}} \\
&\left\langle n_{x}, n_{y}\left|v_{5}\right| n_{x}^{\prime}, n_{y}^{\prime}\right\rangle=-\frac{\tau \hbar^{2}}{2 m} \delta_{n_{x}, n_{x}^{\prime}} \delta_{n_{y}, n_{y}^{\prime}}
\end{aligned}
$$

So the elementes of the perturbation Hamiltonian are given by:

$$
H_{i j}^{\theta, \tau}= \begin{cases}\frac{\tau \hbar^{2}}{4 m} \sqrt{(j-1)(j-2)(g-j+2)(g-j+3)} & ; i-j=-2 \\ \frac{i m \theta \omega^{2}}{2} \sqrt{(j-1)(g-i+1)} & ; i-j=-1 \\ \frac{\tau \hbar^{2}}{4 m}\left[-2(j-1)^{2}+2(j-1)+2(2 j-1) g+2\right] & ; i=j \\ -\frac{i m \theta \omega^{2}}{2} \sqrt{(i-1)(g-j+1)} & ; i-j=1 \\ \frac{\tau \hbar^{2}}{4 m} \sqrt{j(j+1)(g-j+1)(g-j)} & ; i-j=2\end{cases}
$$

where $g=n_{x}+n_{y}$ and integers $i$ and $j$ represent the row and column indices, respectively.

The energy shift for the ground state $n_{x}+n_{y}=0$ is:

$$
\Delta E=-\frac{\tau \hbar^{2}}{2 m}
$$

There is an interesting point involved here, although the correction to the ground state of the harmonic oscillator due to the noncommutativity of space to the 
first order in noncommutativity parameter vanishes in non-dynamical spaces ( $\theta$-noncommutative space ) [5], it has nonvanishing value $-\frac{\tau \hbar^{2}}{2 m}$ in dynamical case. The first excited state $n_{x}+n_{y}=1$, has two fold degeneracy. The perturbation matrix is given by:

$$
\left[\begin{array}{cc}
\frac{\tau \hbar^{2}}{m} & \frac{1}{2} m \omega^{2} \theta \\
-\frac{1}{2} m \omega^{2} \theta & 2 \frac{\tau \hbar^{2}}{m}
\end{array}\right]
$$

and the corresponding energies are:

$$
E_{n_{x}+n_{y}=1}=\left\{\begin{array}{l}
2 \hbar \omega+\frac{3 \tau \hbar^{2}}{2 m}-\sqrt{\frac{\tau^{2} \hbar^{4}}{4 m^{2}}+\frac{m^{2} \omega^{4} \theta^{2}}{4}} \\
2 \hbar \omega+\frac{3 \tau \hbar^{2}}{2 m}+\sqrt{\frac{\tau^{2} \hbar^{4}}{4 m^{2}}+\frac{m^{2} \omega^{4} \theta^{2}}{4}}
\end{array}\right.
$$

\section{Quantum Hall effect}

Quantum Hall effect( QHE ) is the remakable observation of quantized transport in two dimensional electron gasses placed in a transverse magnetic field. Let us consider a moving particle with charge $q$ and mass $\mu$ in a two dimensional $x-y$ plane and submitted to a uniform magnetic field $\vec{B}$ in the $z$-direction. The components of vector potential can be taken as follows:

$$
A_{x}=-\frac{1}{2} B y \quad, \quad A_{y}=\frac{1}{2} B x \quad, \quad A_{z}=0
$$

For the Hamiltonian of the system we have:

$$
H\left(x, y, p_{x}, p_{y}\right)=\frac{1}{2 \mu}\left[\left(p_{x}+\frac{q B}{2 c} y\right)^{2}+\left(p_{y}-\frac{q B}{2 c} x\right)^{2}\right]
$$

In terms of the $\theta$-noncommutative variables, the Hamiltonian takes the following form:

$$
\begin{aligned}
H\left(x_{0}, y_{0}, p_{x_{0}}, p_{y_{0}}\right) & =\frac{1}{2 \mu}\left[\left(p_{x_{0}}+\frac{q B}{2 c} y_{0}\right)^{2}+\left[\left(1+\tau y_{0}^{2}\right)^{1 / 2} p_{y_{0}}\left(1+\tau y_{0}^{2}\right)^{1 / 2}\right.\right. \\
& \left.\left.-\frac{q B}{2 c}\left(1+\tau y_{0}^{2}\right)^{1 / 2} x_{0}\left(1+\tau y_{0}^{2}\right)^{1 / 2}\right]^{2}\right] \\
& =h_{h o}^{\theta}-2 \theta \mu \omega_{L} \tau^{2} \hbar y_{0}^{2}+2 i \omega_{L}\left(\tau y_{0}+\tau^{2} y_{0}^{3}\right)\left[\hbar x_{0}+\theta p_{y_{0}}\right] \\
& -\omega_{L} \tau y_{0}^{2} p_{y_{0}} x_{0}+\tau \hbar \omega_{L} \theta-\omega_{L} l_{z_{0}}
\end{aligned}
$$

where $h_{h o}^{\theta}$ is the Hamiltonian of the harmonic oscillator in $\theta$-noncommutative space, with angular frequency $\omega_{L}=\frac{q B}{2 \mu c}$. In terms of the standard commutative 
space variables it is given by:

$$
\begin{aligned}
H\left(x_{s}, y_{s}, p_{x_{s}}, p_{y_{s}}\right) & =\frac{1}{2 \mu}\left(p_{x_{s}}^{2}+p_{y_{s}}^{2}\right)+\frac{1}{2} \mu \omega_{L}^{2}\left(x_{s}^{2}+y_{s}^{2}\right)-\omega_{L} L_{z_{s}} \\
& -\frac{\mu \omega_{L}^{2} \theta}{2 \hbar} L_{z_{s}}+\frac{\tau}{\mu} y_{s}^{2} p_{y_{s}}^{2}+\mu \omega_{L}^{2} \tau y_{s}^{2} x_{s}^{2}-2 i \frac{\tau \hbar}{\mu} y_{s} p_{y_{s}}-\frac{\tau \hbar^{2}}{2 \mu} \\
& +2 i \omega_{L} \tau \hbar y_{s} x_{s}-\omega_{L} \tau y_{s}^{2} p_{y_{s}} x_{s}+\frac{\omega_{L} \theta}{2 \hbar}\left(p_{x_{s}}^{2}+p_{y_{s}}^{2}\right) \\
& =H_{h o}^{s}+H^{\theta, \tau}
\end{aligned}
$$

It is convenient to write the perturbation Hamiltonian in circular cylinderical coordinates:

$$
\begin{aligned}
H^{\theta, \tau} & =-\frac{\tau \hbar^{2}}{\mu}\left\{\rho^{2} \sin ^{4} \varphi \frac{\partial^{2}}{\partial \rho^{2}}-m^{2} \sin ^{2} \varphi \cos ^{2} \varphi-2 i m \sin ^{3} \varphi \cos \varphi\right. \\
& \left.+2 i m \rho \sin ^{3} \varphi \cos \varphi \frac{\partial}{\partial \rho}\right\}+\mu \omega_{L}^{2} \tau \rho^{4} \sin ^{2} \varphi \cos ^{2} \varphi+\left\{i m \cos ^{2} \varphi\right. \\
& \left.+\rho \sin \varphi \cos \varphi \frac{\partial}{\partial \rho}\right\}-2 \frac{\tau \hbar^{2}}{\mu}\left\{\rho \sin ^{2} \varphi \frac{\partial}{\partial \rho}+i m \sin \varphi \cos \varphi\right\}-\frac{\tau \hbar^{2}}{2 \mu} \\
& +2 i \omega_{L} \tau \hbar \rho^{2} \sin \varphi \cos \varphi+i \hbar \omega_{L} \tau\left\{\sin ^{3} \varphi \cos \varphi \frac{\partial}{\partial \rho}+i m \rho^{3} \sin ^{2} \varphi\right. \\
& \left.\cos ^{2} \varphi\right\}-\hbar \omega_{L} \theta\left\{\sin ^{2} \varphi \frac{\partial^{2}}{\partial \rho^{2}}-\frac{m^{2}}{\rho^{2}} \cos ^{2} \varphi-2 i \frac{m}{\rho^{2}} \sin \varphi \cos \varphi\right. \\
& \left.+2 i \frac{m}{\rho} \sin \varphi \cos \varphi \frac{\partial}{\partial \rho}+\frac{\cos ^{2} \varphi}{\rho} \frac{\partial}{\partial \rho}\right\}
\end{aligned}
$$

Using the wave function:

$$
\Psi_{n_{\rho}, m}(\rho, \varphi)=\sqrt{\frac{\left(n_{\rho}\right) ! \xi^{2|m|+2}}{\pi\left(n_{\rho}+|m|\right) !}} \rho^{|m|} L_{n_{\rho}}^{|m|}\left(\xi^{2} \rho^{2}\right) e^{i m \varphi} e^{-1 / 2 \xi^{2} \rho^{2}} \quad ; \quad \xi^{2}=\frac{\mu \omega_{L}}{\hbar}
$$

we calculate the energy spectrum of the ground state:

$$
E_{m=0, n_{\rho}=0}=\hbar \omega_{L}+\frac{1}{4 \mu \xi^{4}}\left[\tau \mu^{2} \omega_{L}^{2}+\tau \xi^{4} \hbar^{2}+2 \hbar \mu \theta \omega_{L} \xi^{6}\right]=0.25 \frac{\tau \hbar^{2}}{\mu}+\frac{1}{2} \hbar \theta \omega_{L} \xi^{2}
$$

For the first excited state we have:

$$
E_{m=0,1 ; n_{\rho}=1}=3 \hbar \omega_{L}+\left\{\begin{array}{l}
\frac{1}{4 \mu \xi^{4}}\left[7 \tau \mu^{2} \omega_{L}^{2}+7 \tau \xi^{4} \hbar^{2}+6 \hbar \mu \theta \omega_{L} \xi^{6}\right]=1.75 \frac{\tau \hbar^{2}}{\mu}+1.5 \hbar \theta \omega_{L} \xi^{2} \\
\frac{1}{8 \mu \xi^{4}}\left[\left(6 \tau-\theta \xi^{4}\right) \mu^{2} \omega_{L}^{2}+6 \tau \xi^{4} \hbar^{2}+\hbar \mu \omega_{L} \xi^{2}\left(-\tau+4 \theta \xi^{4}\right)\right]=0.7 \frac{\tau \hbar^{2}}{\mu}+ \\
0.5 \hbar \theta \omega_{L} \xi^{2}-0.125 \mu \theta \omega_{L}^{2}
\end{array}\right.
$$


By setting $\tau=0$, we obtaion the results of non-dynamical spaces, for instance Eqs.(22) and (23) give the same values for the energy of the ground and first excited states of the system as Eq.(20) in [6].

\section{Hydrogen atom}

In this section we study the Hydrogen atom in a two dimensional dynamical noncommutative spaces. Electronic bound states around charged impurities in two dimensional systems can be described in terms of a two dimensional Hydrogen atom.

The Hamiltonian of the system is as follows:

$$
H\left(x, y, p_{x}, p_{y}\right)=\frac{1}{2 m}\left(p_{x}^{2}+p_{y}^{2}\right)-\frac{z e^{2}}{\sqrt{x^{2}+y^{2}}}
$$

In terms of the $\theta$-noncommutative variables it takes the following form:

$$
\begin{aligned}
h\left(x_{0}, y_{0}, p_{x_{0}}, p_{y_{0}}\right)= & \frac{1}{2 m}\left[p_{x_{0}}^{2}+\left(1+\tau y_{0}^{2}\right)^{1 / 2} p_{y_{0}}\left(1+\tau y_{0}^{2}\right) p_{y_{0}}\left(1+\tau y_{0}^{2}\right)^{1 / 2}\right] \\
& -z e^{2}\left[\left(1+\tau y_{0}^{2}\right)^{1 / 2} x_{0}\left(1+\tau y_{0}^{2}\right) x_{0}\left(1+\tau y_{0}^{2}\right)^{1 / 2}+y_{0}^{2}\right]^{-1 / 2} \\
= & \frac{1}{2 m}\left[p_{x_{0}}^{2}+\left(1+\tau y_{0}^{2}\right)^{2} p_{y_{0}}^{2}\right]-\frac{2 i \hbar \tau}{m} y_{0}\left(1+\tau y_{0}^{2}\right) p_{y_{0}} \\
& -\frac{\tau^{2} \hbar^{2}}{m} y_{0}^{2}-\frac{\tau \hbar^{2}}{2 \mu}-z e^{2}\left[\left(1+\tau y_{0}^{2}\right)^{2} x_{0}^{2}+4 i \theta \tau y_{0}\right. \\
& \left.\left(1+\tau y_{0}^{2}\right) x_{0}-\tau \theta^{2}-2 \tau^{2} \theta^{2} y_{0}^{2}+y_{0}^{2}\right]^{-1 / 2}
\end{aligned}
$$

In standard commutative space we have:

$$
\begin{aligned}
H\left(x_{s}, y_{s}, p_{x_{s}}, p_{y_{s}}\right) & =\frac{1}{2 m}\left(p_{x_{s}}^{2}+p_{y_{s}}^{2}\right)-\frac{z e^{2}}{\left(x_{s}^{2}+y_{s}^{2}\right)^{1 / 2}}+\frac{z e^{2}}{2\left(x_{s}^{2}+y_{s}^{2}\right)^{3 / 2}} \\
& \times\left[2 \tau x_{s}{ }^{2} y_{s}{ }^{2}-\frac{\theta}{\hbar} L_{z_{s}}\right]+\frac{\tau}{m} y_{s}^{2} p_{y_{s}}^{2}-2 i \frac{\tau \hbar}{m} y_{s} p_{y_{s}}-\frac{\tau \hbar^{2}}{2 m} \\
& =H^{s}+H^{\theta, \tau}
\end{aligned}
$$

If we take $\tau=0$ we obtain $H_{\theta}=-\frac{z e^{2} L_{z} \theta}{2 \hbar r^{3}}$, which is in agreement with the results of non-dynamical case presented in Eq.(2.5) of Ref.[7](note that in [7], $\theta_{i j}$ is defined through $\theta_{i j}=\frac{1}{2} \epsilon_{i j k} \theta_{k}$ but we have chosen $\left.\theta_{i j}=\epsilon_{i j k} \theta_{k}\right)$.

Using the wave functions of two dimentional Hydrogen atom [8], The energy spectrum for the ground state and the first degenerate excited states are obtain:

$$
\triangle E=0.125 \frac{\tau \hbar^{2}}{m}
$$


and

$$
\triangle E=\left\{\begin{array}{l}
0.875 \frac{\tau \hbar^{2}}{2 m} \\
0.75 \frac{\tau \hbar^{2}}{m}-\sqrt{0.06 \frac{\tau^{2} \hbar^{4}}{m^{2}}+0.04 \frac{\left(z e^{2}\right)^{2} \theta^{2}}{a_{0}^{6}}} \\
0.75 \frac{\tau \hbar^{2}}{m}+\sqrt{0.06 \frac{\tau^{2} \hbar^{4}}{m^{2}}+0.04 \frac{\left(z e^{2}\right)^{2} \theta^{2}}{a_{0}^{6}}}
\end{array}\right.
$$

\section{The Zeeman effect}

The Zeeman effect is very important in applications such as nuclear magnetic resonance spectroscopy, electron spin resonance spectroscopy, magnetic resonance imaging ( MRI ) and Mössbaure spectroscopy. To study the effects of dynamical noncommutativity on Zeeman effect we consider the relevant Hamiltonian:

$$
\begin{aligned}
H\left(x, y, p_{x}, p_{y}\right) & =\frac{1}{2 m}\left(p_{x}^{2}+p_{y}^{2}\right)-\frac{z e^{2}}{\sqrt{x^{2}+y^{2}}}-\omega L_{z} \\
& =\frac{1}{2 m}\left(p_{x}^{2}+p_{y}^{2}\right)-\frac{z e^{2}}{\sqrt{x^{2}+y^{2}}}-\omega\left[x p_{y}-y p_{x}\right]
\end{aligned}
$$

In terms of the $\theta$-noncommutative variables it takes the following form:

$$
\begin{aligned}
& H\left(x_{0}, y_{0}, p_{x_{0}}, p_{y_{0}}\right)=\frac{1}{2 m}\left[p_{x_{0}}^{2}+\left(1+\tau y_{0}^{2}\right)^{2} p_{y_{0}}^{2}\right]-\frac{2 i \hbar \tau}{m} y_{0}\left(1+\tau y_{0}^{2}\right) p_{y_{0}} \\
&-\frac{\tau^{2} \hbar^{2}}{m} y_{0}^{2}-\frac{\tau \hbar^{2}}{2 m}-z e^{2}\left[\left(1+\tau y_{0}^{2}\right)^{2} x_{0}^{2}+4 i \theta \tau\right. \\
&\left.y_{0}\left(1+\tau y_{0}^{2}\right) x_{0}-\tau \theta^{2}-2 \tau^{2} \theta^{2} y_{0}^{2}+y_{0}^{2}\right]^{1 / 2}-\omega \\
& {\left[\left(1+\tau y_{0}^{2}\right)^{1 / 2} x_{0}\left(1+\tau y_{0}^{2}\right) p_{y_{0}}\left(1+\tau y_{0}^{2}\right)^{1 / 2}-y_{0} p_{x_{0}}\right] }
\end{aligned}
$$

Using Bopp-shift one can write down the Hamiltonian in standard commutative

space:

$$
\begin{aligned}
H\left(x_{s}, y_{s}, p_{x_{s}}, p_{y_{s}}\right) & =\frac{1}{2 m}\left(p_{x_{s}}^{2}+p_{y_{s}}^{2}\right)-\frac{z e^{2}}{\left(x_{s}^{2}+y_{s}^{2}\right)^{1 / 2}}+\frac{z e^{2}}{2\left(x_{s}^{2}+y_{s}^{2}\right)^{3 / 2}} \\
& \times\left[2 \tau x_{s}{ }^{2} y_{s}{ }^{2}-\frac{\theta}{\hbar} L_{z_{s}}\right]+\frac{\tau}{m} y_{s}^{2} p_{y_{s}}^{2}-2 i \frac{\tau \hbar}{m} y_{s} p_{y_{s}}-\frac{\tau \hbar^{2}}{2 m} \\
& -\omega\left[x_{s} p_{y_{s}}-y_{s} p_{x_{s}}\right]+\frac{\theta}{2 \hbar} \omega\left(p_{x_{s}}^{2}+p_{y_{s}}^{2}\right) \\
& -2 \tau \omega y_{s}^{2} p_{y_{s}} x_{s}+i \hbar \omega \tau y_{s} x_{s}
\end{aligned}
$$


If we choose $\tau=0$, Eq.(31) reduces to the Eqs.(24), (25) and (26) of the Ref.[9] for the case of non-dynamical spaces(to the first order in magnetic field $B$ ). The corrections to the energy for the ground state and degenerate first excited state are given by:

$$
\triangle E=2 \frac{\hbar \omega \theta}{a_{0}^{2}}+0.125 \frac{\tau \hbar^{2}}{m}
$$

and

$$
\triangle E=\left\{\begin{array}{l}
0.22 \frac{\hbar \omega \theta}{a_{0}^{2}}+0.875 \frac{\tau \hbar^{2}}{m} \\
\frac{0.5}{a_{0}^{6} m^{2}}\left[-a_{0}^{4} \hbar m\left(-1.5 a_{0}^{2} \tau \hbar-0.4 m \theta \omega\right)-0.5 a_{0}^{3}\left(a_{0}^{6} \hbar^{4} m^{2} \tau^{2}\right.\right. \\
\left.\left.+0.64 m^{4}\left(z e^{2}\right)^{2} \theta^{2}-17.92 z e^{2} a_{0}^{5} \hbar m^{4} \tau \theta \omega\right)^{1 / 2}\right]= \\
0.75 \frac{\tau \hbar^{2}}{m}+0.2 \frac{\hbar \theta \omega}{a_{0}^{2}}-0.5\left[\left(\frac{\tau \hbar^{2}}{m}\right)^{2}-17.92 \frac{\tau \hbar^{2}}{m} \frac{\left(z e^{4}\right) \theta \omega m^{2}}{\hbar^{3}}+0.64 \frac{\left(z e^{2}\right)^{2} \theta^{2}}{a_{0}^{6}}\right] \frac{1}{2} \\
\frac{0.5}{a_{0}^{6} m^{2}}\left[-a_{0}^{4} \hbar m\left(-1.5 a_{0}^{2} \tau \hbar-0.4 m \theta \omega\right)+0.5 a_{0}^{3}\left(a_{0}^{6} \hbar^{4} m^{2} \tau^{2}\right.\right. \\
\left.+0.64 m^{4}\left(z e^{2}\right)^{2} \theta^{2}-17.92 z e^{2} a_{0}^{5} \hbar m^{4} \tau \theta \omega\right)^{1 / 2}= \\
0.75 \frac{\tau \hbar^{2}}{m}+0.2 \frac{\hbar \theta \omega}{a_{0}^{2}}+0.5\left[\left(\frac{\tau \hbar^{2}}{m}\right)^{2}-17.92 \frac{\tau \hbar^{2}}{m} \frac{\left(z e^{4}\right) \theta \omega m^{2}}{\hbar^{3}}+0.64 \frac{\left(z e^{2}\right)^{2} \theta^{2}}{a_{0}^{6}}\right] \frac{1}{2}
\end{array}\right.
$$

where $\omega=\frac{e B}{2 \mu c}$ and $a_{0}$ is the Bohr radius.

\section{The Stark effect}

Stark effect has become an increasingly important part of atomic, molecular and optical physics. To study the effects of the external electric field on the spectrum of the Hydrogen atom in dynamical noncommutative spaces, we consider the electric field in the $x$-direction. The Hamiltonian is

$$
H\left(x, y, p_{x}, p_{y}\right)=\frac{1}{2 m}\left(p_{x}^{2}+p_{y}^{2}\right)-\frac{z e^{2}}{\sqrt{x^{2}+y^{2}}}+e E x
$$

For the ground state we have :

$$
\triangle E=0.125 \frac{\tau \hbar^{2}}{m}
$$

It can be shown that for the first excited state the energy correction to the first order is also proportional to $\frac{\tau \hbar^{2}}{m}$.

The same results are obtained if the electric field applied in the $y$-direction.

It is shown in [7] that at tree level the contribution to the Stark effect is zero in non-dynamical case but we observe that in dynamical noncommutative spaces 
the contribution to the Stark effect is nonzero, therefore the Stark effect can be employed to determine whether the noncommutativity of space is dynamical or nondynamical. it is instructive to understand the mechanism intuitively. As we already mentioned the energy correction due to the external electric field on the energy spectrum of the Hydrogen ground state in dynamical noncommutative space is not zero. This means that there is a permanent electric dipole for the ground state of the hydrogen atom in DNCS. To understand how this electric dipole is produced, we note that the fundamental objects in DNCS are not point like but they are string like. On the other hand it is shown in [10] that strings can act as electric dipoles, so the fundamental particles(strings) in dynamical noncommutative spaces for instance electrons, quarks (neurons and proto ns) can have electric dipoles due to dynamical noncommutativity of space which leads to permanent electic dipole for the ground state of the Hydrogen atom.

\section{Conclusions}

We have studied some fundamental and interesting quantum systems in dynamical noncommutative spaces. The energy shift for the ground state of the harmonic oscillator due to noncommutativity of space is zero for non-dynamical case while it has non-vanishing value in DNCS. We have shown that Stark effect can be used to check whether the noncommutativity of space is dynamical or non-dynamical. It seems that in the dynamical noncommutative space introduced here, there is a fundamental energy so that the corrections due to dynamical noncommutativity on the energy of a quantum system can be stated in terms of $g \frac{\tau \hbar^{2}}{m}$ in which $\frac{\tau \hbar^{2}}{m}$ is fundamental enegy independent of the system and the factor $g$ depends on the kind of the system. For instance, for the ground state of the harmonic oscillator and quantum Hall effect, the values of $g$ are -0.5 and 0.25 respectively. For Hydrogen atom, Zeeman and stark effects it is 0.125 . We confirm that in the limit $\tau \rightarrow 0$, we obtain the results presented in previous works devoted to non-dynamical noncommutative spaces. Finally one can use the energy accuracy measurement $10^{-12} \mathrm{eV}$ [11] to impose some bounds on the value of the noncommutativity parameter $\tau: \frac{\tau \hbar^{2}}{m}<10^{-12} \mathrm{eV}$.

\section{References}

[1] N. Seiberg and E. Witten;" String theory and noncommutative geometry" JHEP 9909: 032, (1999); arXiv:hep-th/9908142.

[2] F. Ardalan, H. Arfaei and M. M. Sheikh-Jabbari;" Noncommutative geometry from strings and branes" JHEP 9902 (1999) 016; arXiv:hep-th/9810072.

[3] M. Gomes, V. G. Kupriyanov;" Position-dependent noncommutativity in quantum mechanics" Phys. Rev. D 79 (2009) 125011. M. Gomes, V. G. Kupriyanov, A. J. da Silva;" Dynamical noncommutativity" J. Phys. A 43 (2010) 285301. 
[4] A. Fring, L. Gouba and F. G. Scholtz;" Strings from position-dependent noncommutativity" J. Phys. A: Math. Theor. 43 (2010) 345401; [hep-th/1003.3025].

[5] A. Jahan;" Noncommutative Harmonic Oscillator at Finite Temperature: A Path Integral Approach" Braz. J. Phys. 38 (2008) 144; [ arXiv:1208.0137].

[6] S. Dulat and K. Li;" Landau problem in noncommutative quantum mechanics" Chin. Phys. C 32 (2008) 92.; [math-ph/0802.1118]

[7] M. Chaichian, M. M. Sheikh-Jabbari, A. Tureanu ;" Hydrogen Atom Spectrum and the Lamb Shift in Noncommutative QED" Phys.Rev.Lett.86 (2001) 2716; [hepth/0010175].

[8] J. S. Dehesa, S. Lopez-Rosa, B. Olmos and R. J. Yanes;" Fisher information of D-dimensional hydrogenic systems in position and momentum spaces" J. Math. Phys.47 (2006) 052104 .

[9] W. O. Santos, A. M. C. Souza;" The anomalous Zeeman effect for the Hydrogen atom in noncommutative space" Int. J. Theor. Phys. 51 (2012) 3882.

[10] M. M. Sheikh Jabbari;" Open Strings in a B-field Background as Electric Dipoles" Phys.Lett.B 455 (1999) 129; hep-th/9901080.

[11] Essen et.al., Nature 229(1971)110. 